\title{
The management of active surveillance in prostate cancer: validation of the Canary Prostate Active Surveillance Study risk calculator with the Spanish Urological Association Registry
}

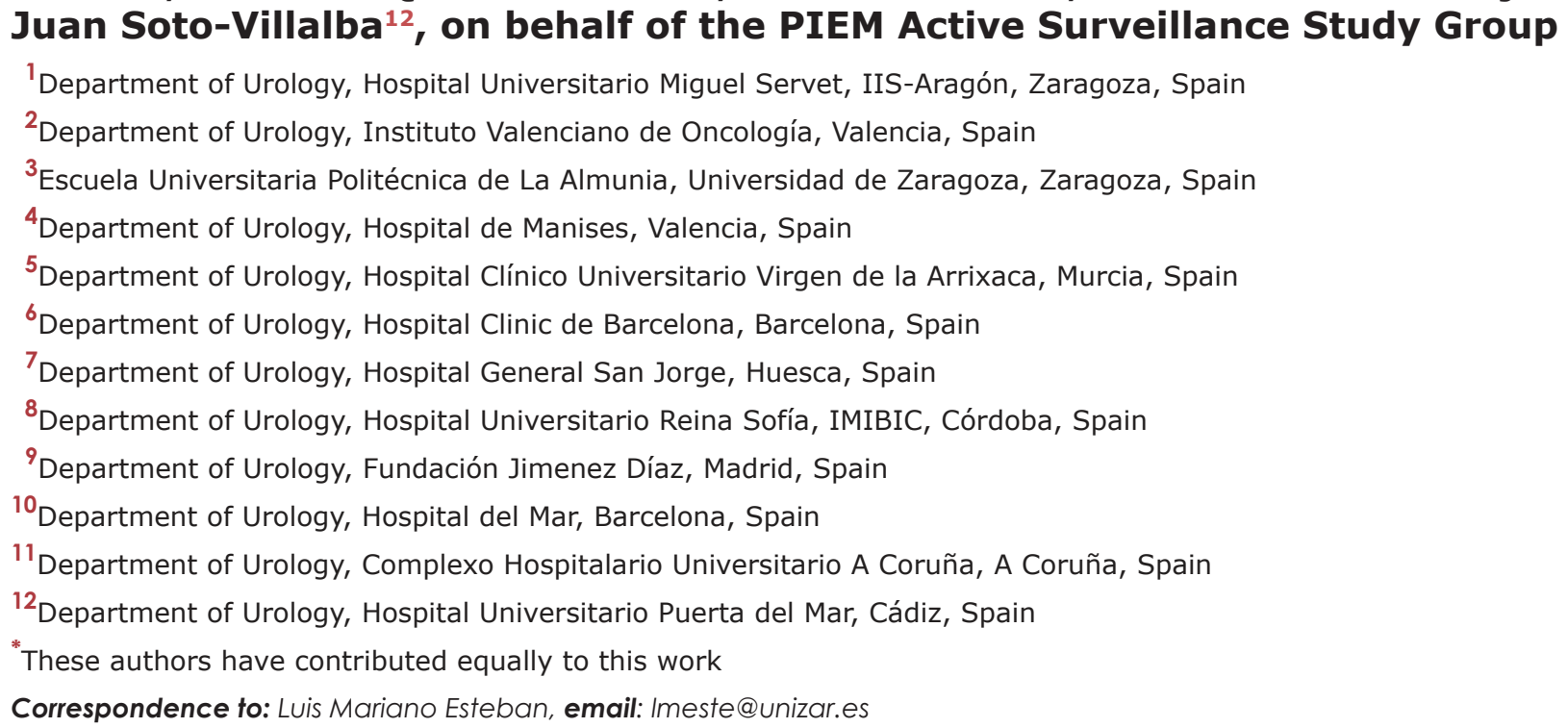
Juan Soto-Villalba ${ }^{12}$, on behalf of the PIEM Active Surveillance Study Group

${ }^{1}$ Department of Urology, Hospital Universitario Miguel Servet, IIS-Aragón, Zaragoza, Spain

${ }^{2}$ Department of Urology, Instituto Valenciano de Oncología, Valencia, Spain

${ }^{3}$ Escuela Universitaria Politécnica de La Almunia, Universidad de Zaragoza, Zaragoza, Spain

${ }^{4}$ Department of Urology, Hospital de Manises, Valencia, Spain

${ }^{5}$ Department of Urology, Hospital Clínico Universitario Virgen de la Arrixaca, Murcia, Spain

${ }^{6}$ Department of Urology, Hospital Clinic de Barcelona, Barcelona, Spain

${ }^{7}$ Department of Urology, Hospital General San Jorge, Huesca, Spain

${ }^{8}$ Department of Urology, Hospital Universitario Reina Sofía, IMIBIC, Córdoba, Spain

${ }^{9}$ Department of Urology, Fundación Jimenez Díaz, Madrid, Spain

${ }^{10}$ Department of Urology, Hospital del Mar, Barcelona, Spain

${ }^{11}$ Department of Urology, Complexo Hospitalario Universitario A Coruña, A Coruña, Spain

${ }^{12}$ Department of Urology, Hospital Universitario Puerta del Mar, Cádiz, Spain

*These authors have contributed equally to this work

Correspondence to: Luis Mariano Esteban, email: Imeste@unizar.es Collado-Serra², Yoni Pallás-Costa ${ }^{4}$, Pedro Ángel López-González ${ }^{5}$ Jorge HuguetPérez $^{6}$, José Ignacio Sanz-Vélez ${ }^{7}$, Jesús Manuel Gil-Fabra ${ }^{1}$, Enrique GómezGómez $^{8}$, Cristina Quicios-Dorado9, Lluis Fumadó ${ }^{10}$, Sara Martínez-Breijo ${ }^{11}$ and

Keywords: active surveillance; prostate cancer; reclassification; risk calculator; external validation Received: August 10, $2017 \quad$ Accepted: October 03, $2017 \quad$ Published: October 24, 2017

Copyright: Borque-Fernando et al. This is an open-access article distributed under the terms of the Creative Commons Attribution License 3.0 (CC BY 3.0), which permits unrestricted use, distribution, and reproduction in any medium, provided the original author and source are credited.

\section{ABSTRACT}

The follow up of patients on active surveillance requires to repeat prostate biopsies. Predictive models that identify patients at low risk of progression or reclassification are essential to reduce the number of unnecessary biopsies. The aim of this study is to validate the Prostate Active Surveillance Study risk calculator (PASS-RC) in the multicentric Spanish Urological Association Registry of patients on active surveillance (AS), from common clinical practice.

Results: We find significant differences in age, PSA and clinical stage between our validation cohort and the PASS-RC generation cohort $(p<.0001)$, with a reclassification rate of $10-22 \%$ on the follow-up $B x$, no cancer was found in $43 \%$ of the first follow-up Bx. The calibration curve shows underestimation of real appearance of reclassification. The AUC is 0.65 (C.I.95\%: 0.60-0.71). PDF and CUC do not suggest a specific cut-off point of clinical use.

Methods: We select 498 patients on AS with a minimum of one follow-up biopsy (Bx) from the 1,024 males registered by 36 Spanish centers recruiting patients on the Spanish Urological Association Registry on AS. PASS-RC external validation is carried by means of calibration curve and area under de ROC-curve (AUC), identifying 


\section{cut-offs of clinical utility by probability density functions (PDF) and clinical utility curves (CUC). \\ Conclusions: In our first external validation of the PASS-RC we have obtained a moderate discrimination ability, although we cannot recommend cut-off points of clinical use. We suggest the exploration of new biomarkers and/or morpho-functional parameters from multiparametric magnetic resonance image, to improve those necessary tools on AS.}

\section{INTRODUCTION}

Active surveillance (AS) is increasingly being implemented by urologists as a strategy that provides the benefits from prostate cancer (PCa) opportunistic screening observed in western countries [1], but avoids overtreatment. We have presented our National Registry (AEU/PIEM/2014/0001, www.piem.aeu.es) supported by the Spanish Urological Association (Asociación Española de Urología, AEU) [2]. This initiative was created with the aim of facilitating the implementation of AS in all types of Hospitals, and of providing an opportunity for multicentric clinical research, as different inclusion criteria and followup strategies are allowed. These wider inclusion criteria are contemplated in other AS registers such as the Canary Prostate Active Surveillance Study (PASS) [3], in contrast with the more strict inclusion criteria used by previous series [4-10].

All the different AS protocols coincide on the necessity to perform repeated prostatic biopsies $(\mathrm{Bx})$ which are not free of complications [11], with increasing antibiotic resistance as a major problem. Recently, the initial results of the PASS study (clinicaltrials.gov NCT00756665) have been published, with a median follow-up of 28 months: $24 \%$ of their patients faced adverse reclassification [3]. These data are frequently found in a current database like ours [2] among others $[12,13]$, all of which have been previously driven by the uncertainty of the common selection criteria used. Using a cohort of 859 Gleason 6 PCa patients, in their late study the same group has proposed an easy to use online prediction tool [14] of progression in AS, with widely used and reproducible covariates. This tool showed an area under the curve (AUC) of $72.4 \%$ for reclassification in the follow-up Bx, providing an on-line tool to facilitate counselling for the patients on AS [15].

Although new biomarkers and data from multiparametric Magnetic Resonance Imaging (mpMRI) could improve its predictive accuracy [16-19], the real strength of this new tool relies on its variables: age, PSA, months from last biopsy, percentage of positive cores for $\mathrm{PCa}$ on the last $\mathrm{Bx}$ and number of prior negative $\mathrm{Bx}$; all of them available and reproducible elsewhere. Both criteria, new biomarkers such as PCA3 and PHI [20] and mpMRI [21], have shown their ability for a better selection of patients for AS when referred to pathological results of radical prostatectomy specimens, but they will need to demonstrate clear advantages in gaining accuracy, but also their cost-effectiveness, if they are to be regularly introduced in AS protocols. Without mpMRI and new biomarkers and using just the criteria utilized in this model, the long term results of the AS series show excellent disease-free survival results [19].

As the PASS and AEU protocols are comparable in their design $[2,3]$, we aim to perform an external validation of this PASS-RC with a different population. We understand that our external validation study specially focused on defining clinical utility of PASS-RC could make this tool a useful help for decision making in men on AS.

\section{RESULTS}

From the 1,024 Prostate cancer patients recruited in the PIEM cohort until December 31st 2015, only 498 of them had at least one follow-up Bx to evaluate the possibility of Bx reclassification by the PASS-RC. These 498 patients form the validation set and come from 24 Spanish hospitals, the median follow-up was 19.7 months with $25 \%-75 \%$ percentiles of 11.4 and 33.7 months, respectively. Among them, the patients without reclassification had a median follow-up until last biopsy of 11.7 months, with P25-75 of 7.2-18.3. The median time between reclassification and first biopsy was 9.5 months with P25-75 of 7-16.7 months. We observed statistically significant differences for the distribution of variables age, race, PSA and T stage at diagnosis between both cohorts (Table 1).

Our cohort had a maximum of four follow-up Bx (Table 2), with slightly superior patient mean age at the different biopsies. The follow-up Bx had been performed at a mean of 2 months before in the AEU cohort. The mean PSA value was approximately $1 \mathrm{ng} / \mathrm{ml}$ greater in our series. The number of biopsy cores had the same mean 12 cores for the diagnostic biopsy at both series, but our validation cohort practiced significantly more cores at the follow-up-Bx.

The analysis of the variables that define reclassification shown in Figure 1 illustrates the percentages of reclassification for the PIEM and PASS database at the four follow-up biopsies. The calculated $p$-values of $0.92,0.23,0.12$ and 0.99 respectively also reflect the equivalence between percentages of reclassification in all follow-up biopsies.

As regards the validation of the PASS-RC, we obtained the calibration curve that shows an 
Table 1: Descriptive characteristics of PASS cohort versus PIEM-AEU- AS validation series

\begin{tabular}{|c|c|c|c|c|c|c|}
\hline \multirow{2}{*}{ Variable } & & \multicolumn{2}{|c|}{ PIEM-AEU-AS $(N=498)$} & \multicolumn{2}{|c|}{$\operatorname{PASS}(N=859)$} & \multirow{2}{*}{$p$-value } \\
\hline & & $N$ & $\%$ & $N$ & $\%$ & \\
\hline \multicolumn{7}{|c|}{ Age at diagnosis } \\
\hline & $\leq 49$ & 5 & $1,00 \%$ & 40 & $4,66 \%$ & $<0,001$ \\
\hline & $50-59$ & 102 & $20,48 \%$ & 251 & $29,22 \%$ & \\
\hline & $60-69$ & 248 & $49,80 \%$ & 461 & $53,67 \%$ & \\
\hline & $70-79$ & 143 & $28,71 \%$ & 106 & $12,34 \%$ & \\
\hline & $\geq 80$ & 0 & $0,00 \%$ & 1 & $0,12 \%$ & \\
\hline \multicolumn{7}{|l|}{ Race } \\
\hline & White & 460 & $92,37 \%$ & 783 & $91,15 \%$ & $<0,001$ \\
\hline & Black & 1 & $0,20 \%$ & 43 & $5,01 \%$ & \\
\hline & Other-NA & 37 & $7,43 \%$ & 33 & $3,84 \%$ & \\
\hline \multicolumn{7}{|c|}{$\begin{array}{l}\text { PSA at diagnosis, } \\
\mathrm{ng} / \mathrm{mL}\end{array}$} \\
\hline & $0-2.5$ & 11 & $2,21 \%$ & 104 & $12,11 \%$ & $<0,001$ \\
\hline & $2.5-4$ & 83 & $16,67 \%$ & 148 & $17,23 \%$ & \\
\hline & $4-6$ & 193 & $38,76 \%$ & 352 & $40,98 \%$ & \\
\hline & $6-10$ & 176 & $35,34 \%$ & 192 & $22,35 \%$ & \\
\hline & $>10$ & 35 & $7,03 \%$ & 53 & $6,17 \%$ & \\
\hline & NA & 0 & $0,00 \%$ & 10 & $1,16 \%$ & \\
\hline \multicolumn{7}{|c|}{$\mathrm{T}$ stage at diagnosis } \\
\hline & T1a-c & 466 & $93,57 \%$ & 763 & $88,82 \%$ & $<0,001$ \\
\hline & $\mathrm{T} 2 \mathrm{a}$ & 27 & $5,42 \%$ & 92 & $10,71 \%$ & \\
\hline & $\mathrm{T} 2 \mathrm{~b}-\mathrm{c}$ & 1 & $0,20 \%$ & 4 & $0,47 \%$ & \\
\hline & $\mathrm{Na}$ & 4 & $0,80 \%$ & 0 & $0,00 \%$ & \\
\hline
\end{tabular}

underestimation of reclassification probabilities (Figure 2). The ROC curve resulted in an area under the ROC curve (AUC) of 0.65 , lower than the 0.72 obtained in the generation cohort [15] (Figure 3). No statistically significant differences appear in a comparison between Gleason $3+4(\mathrm{AUC}=0.66)$ and Gleason $4+3(\mathrm{AUC}=$ 0.63 ) reclassifications, $p$-value $=0.55$.

Figure 4 shows the probability density function (PDFs) analysis performed. We can see the probabilities of reclassification provided by PASS-RC for the patients who progressed, or who did not, in the PIEM validation cohort, which proves the impossibility to identify a good discriminative cut-off to be recommended for clinical use. The Clinical utility curve (CUC), illustrated in Figure 5, highlights the real clinical impact of these findings. Selecting different threshold reclassification probabilities under those we should not indicate a follow-up Bx, we project this value over the two curves in the graphic.
In one of them, the red one, we can see the percentage of saved follow up-Bx and in a parallel fashion, in the blue curve, the rate of missed reclassifications. Probably, the best threshold corresponds to $13 \%$, which corresponds to a $23 \%$ of potentially saved biopsies at a cost of missing $13 \%$ reclassifications. Alternatively, in a more conservative way, a cut-off of $12 \%$, drives us to a $16 \%$ of saved biopsies and a $6 \%$ of undetected reclassifications (Table 3 , Figure 5). The decision curve, shown in Figure 6, assessed this analysis, there is a narrow range between $18 \%-38 \%$ where PASS-RC have utility, but with a poor net benefit below $10 \%$.

In addition, we have explored the performance of the combination of PASS-RC with the predictors BMI, PSA density, $\mathrm{PCa}$ length in positive cores and the percentage of $\mathrm{PCa}$ involvement in those cores. Results are shown in Table 4. Only PSA density increase significantly the AUC from 0.654 to 0.694 ( $p=0.04)$. 
Table 2: Biopsy characteristics at diagnosis and at each sequential surveillance biopsy

\begin{tabular}{|c|c|c|c|c|c|c|c|c|c|c|}
\hline \multirow{3}{*}{ Characteristics } & \multicolumn{10}{|c|}{ Biopsy } \\
\hline & \multicolumn{2}{|c|}{ Diagnosis } & \multicolumn{2}{|c|}{ First } & \multicolumn{2}{|c|}{ Second } & \multicolumn{2}{|c|}{ Third } & \multicolumn{2}{|c|}{ Fourth } \\
\hline & PIEM & PASS & PIEM & PASS & PIEM & PASS & PIEM & PASS & PIEM & PASS \\
\hline Patients, $n$ & 1024 & 979 & 498 & 859 & 100 & 458 & 20 & 211 & 9 & 75 \\
\hline $\begin{array}{l}\text { Age at biopsy, yr, } \\
\text { mean (SD) }\end{array}$ & $66.1(6.9)$ & $62.0(6.9)$ & $66.5(7.0)$ & $63.0(7.0)$ & $66.4(6.8)$ & $64.4(6.9)$ & $66.2(6.1)$ & $65.5(6.9)$ & $67.1(4.8)$ & $65.5(7.2)$ \\
\hline $\begin{array}{l}\text { Months since last } \\
\text { biopsy, no, mean } \\
\text { (SD) }\end{array}$ & $0(0.0)$ & $0(0.0)$ & $10.9(8.9)$ & $12.8(8.8)$ & $18.4(8.5)$ & $19.7(8.3)$ & $18.9(8.5)$ & $21.1(7.9)$ & $18.0(10.0)$ & $20.1(7.8)$ \\
\hline $\begin{array}{l}\text { Most recent PSA, } \\
\mathrm{ng} / \mathrm{ml} \text {, mean (SD) }\end{array}$ & $6.6(3.0)$ & $5.5(3.0)$ & $6.2(3.5)$ & $5.0(3.3)$ & $6.3(3.6)$ & $5.2(3.6)$ & $6.1(4.3)$ & $5.4(3.6)$ & $8.8(6.1)$ & $5.5(3.9)$ \\
\hline $\begin{array}{l}\text { No. of biopsy } \\
\text { cores, median } \\
\text { (range) }\end{array}$ & $12(10-70)$ & $12(4-60)$ & $16(10-67)$ & $12(4-60)$ & $\begin{array}{c}16 \\
(10-31)\end{array}$ & $12(4-46)$ & $\begin{array}{c}18 \\
(10-24)\end{array}$ & $12(4-46)$ & $20(12-27)$ & $12(4-34)$ \\
\hline \multicolumn{11}{|l|}{$\begin{array}{l}\text { Percentage of } \\
\text { cores positives for } \\
\text { cancer, } n(\%)\end{array}$} \\
\hline 0 & $0(0.0)$ & $0(0.0)$ & $218(43.8)$ & $312(36.3)$ & $49(49)$ & $\begin{array}{c}190 \\
(41.5)\end{array}$ & $10(50)$ & $88(41.7)$ & $4(44.45)$ & $30(40.0)$ \\
\hline$>0$ and $<34$ & $\begin{array}{c}1014 \\
(99.0)\end{array}$ & $860(87.8)$ & $251(50.4)$ & $465(54.1)$ & $44(44)$ & $\begin{array}{c}224 \\
(48.9)\end{array}$ & $8(40)$ & $(49.3)$ & $4(44.45)$ & $41(54.7)$ \\
\hline$\geq 34$ & $6(0.6)$ & $37(3.8)$ & $29(5.8)$ & $66(7.7)$ & $7(7)$ & $37(8.1)$ & $2(10)$ & $18(8.5)$ & $1(11.1)$ & $4(5.3)$ \\
\hline NA & $4(0.4)$ & $82(8.4)$ & - & $16(1.9)$ & - & 7 (1.5) & - & $1(0.5)$ & - & $0(0.0)$ \\
\hline \multicolumn{11}{|l|}{$\begin{array}{l}\text { Gleason score, } \mathrm{n} \\
(\%)\end{array}$} \\
\hline$\leq 6$ & $1024(100)$ & $\begin{array}{c}979 \\
(100.0)\end{array}$ & $413(82.9)$ & $732(85.2)$ & $83(83)$ & $\begin{array}{c}384 \\
(83.8)\end{array}$ & $19(95)$ & $\begin{array}{c}166 \\
(78.7)\end{array}$ & $7(77.8)$ & $63(84.0)$ \\
\hline 7 & $0(0)$ & $0(0.0)$ & $76(15.3)$ & $123(14.3)$ & $13(13)$ & $70(15.3)$ & $1(5)$ & $43(20.4)$ & $2(22.2)$ & $10(13.3)$ \\
\hline$\geq 8$ & $0(0)$ & $0(0.0)$ & $9(1.8)$ & $4(0.5)$ & $4(4)$ & $4(0.9)$ & 0() & $2(1.0)$ & 0() & $2(2.7)$ \\
\hline \multicolumn{11}{|l|}{ Outcome } \\
\hline Reclassification & $0(0)$ & $0(0.0)$ & 94 (18.9) & $163(19.0)$ & $17(17)$ & $\begin{array}{c}104 \\
(22.7)\end{array}$ & $2(10)$ & $61(28.9)$ & $2(22.2)$ & $19(25.3)$ \\
\hline Stable & $1024(100)$ & 897 (91.6) & $404(81.1)$ & $684(79.6)$ & $83(83)$ & $\begin{array}{c}347 \\
(75.8)\end{array}$ & $18(90)$ & $\begin{array}{c}149 \\
(70.6)\end{array}$ & $7(77.8)$ & $56(74.6)$ \\
\hline NA & - & $82(8.4)$ & & $12(1.4)$ & & $7(1.5)$ & & $1(0.5)$ & & $0(0.0)$ \\
\hline
\end{tabular}

\section{DISCUSSION}

Active surveillance is becoming more and more common in our clinical practice. The unavoidable consequences of over-detection in $\mathrm{PCa}$ are not synonymous of over-treatment because AS contributes to a responsible management of patients in low or very low grade and low volume PCa [23]. Nevertheless, still many urologists are reluctant to offer AS to their patients [24], arguing a lack of confidence in conservative management instead of an active treatment, and due to uncertainties with their general pathologists and radiologists with no specific training in mpMRI, or lack of strong evidence that new biomarkers could help in this setting [23]. Other non scientific reasons could be explained by the need to justify economical and technical investments to their health care providers.

Above these arguments, we can mention the accumulative evidence of AS efficiency in PCa control of series with long follow up [22], showing cancer specific survival rates of $99.9 \%$ at 15 years with strictest criteria [25]. This strong argument minimizes the potential benefit in cancer specific survival of active treatment supporters. However, the serious implications of AS should be carefully explained to the patients. Given the urge for evolution, protocols are now trying to become riskadapted. Yet, a follow-up Bx is not free of complications and it can become a clearly stressful episode and a source for discomfort that patients will need to confront. With this aim, protocols will need to be simplified due to the potential huge amount of AS candidates [26, 27], and the predicted costly work overload for Urology Departments.

Is in this context, the PASS initiative for developing an Active Surveillance Biopsy Risk Calculator of Reclassification/Upgrade should be considered as extremely convenient [15]. Previous studies have tried to associate PSA kinetics [28], number of previous positive 


\section{Progression in Follow-up biopsies}

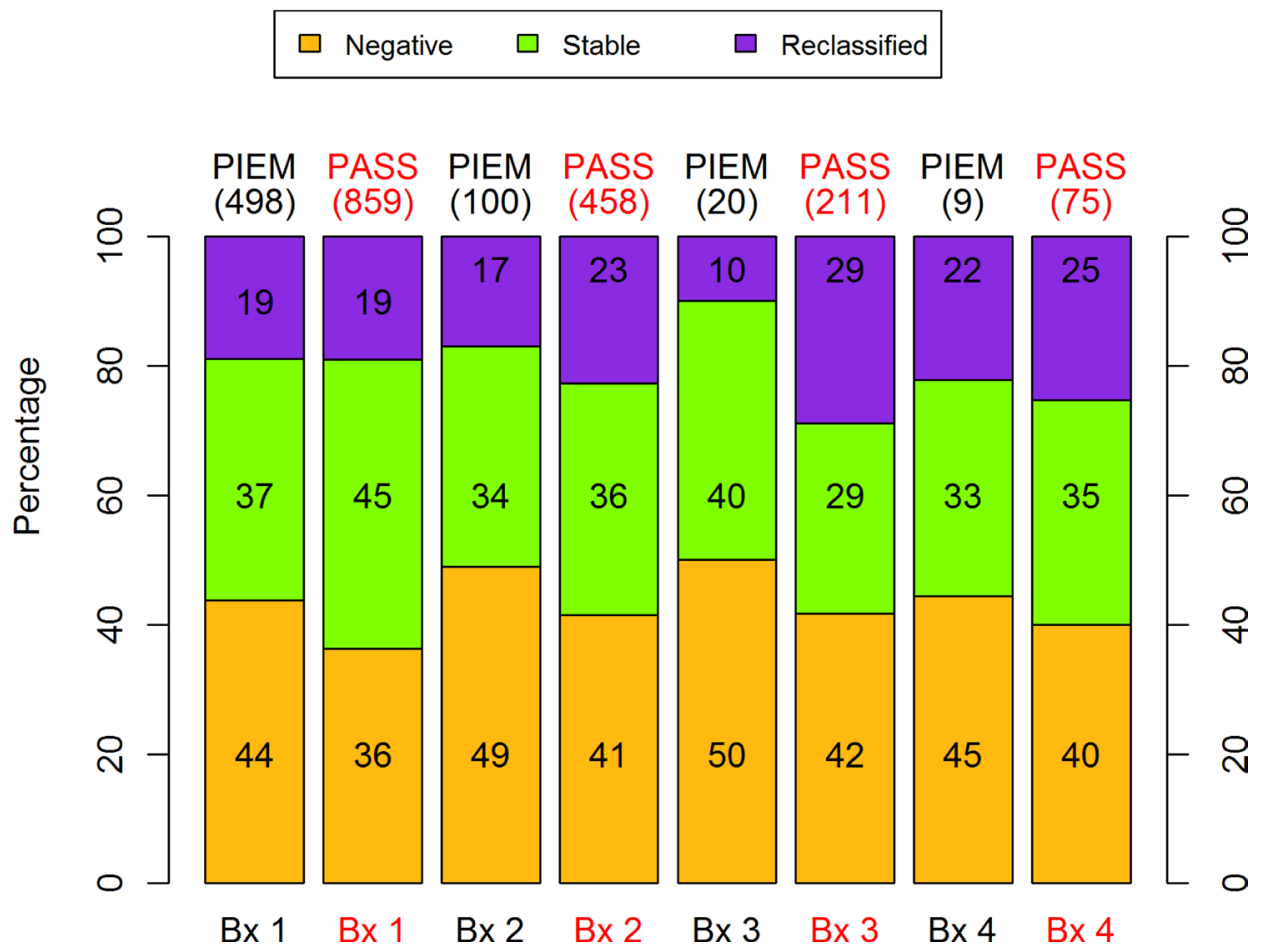

Figure 1: Percentage of reclassification in follow-up biopsies.

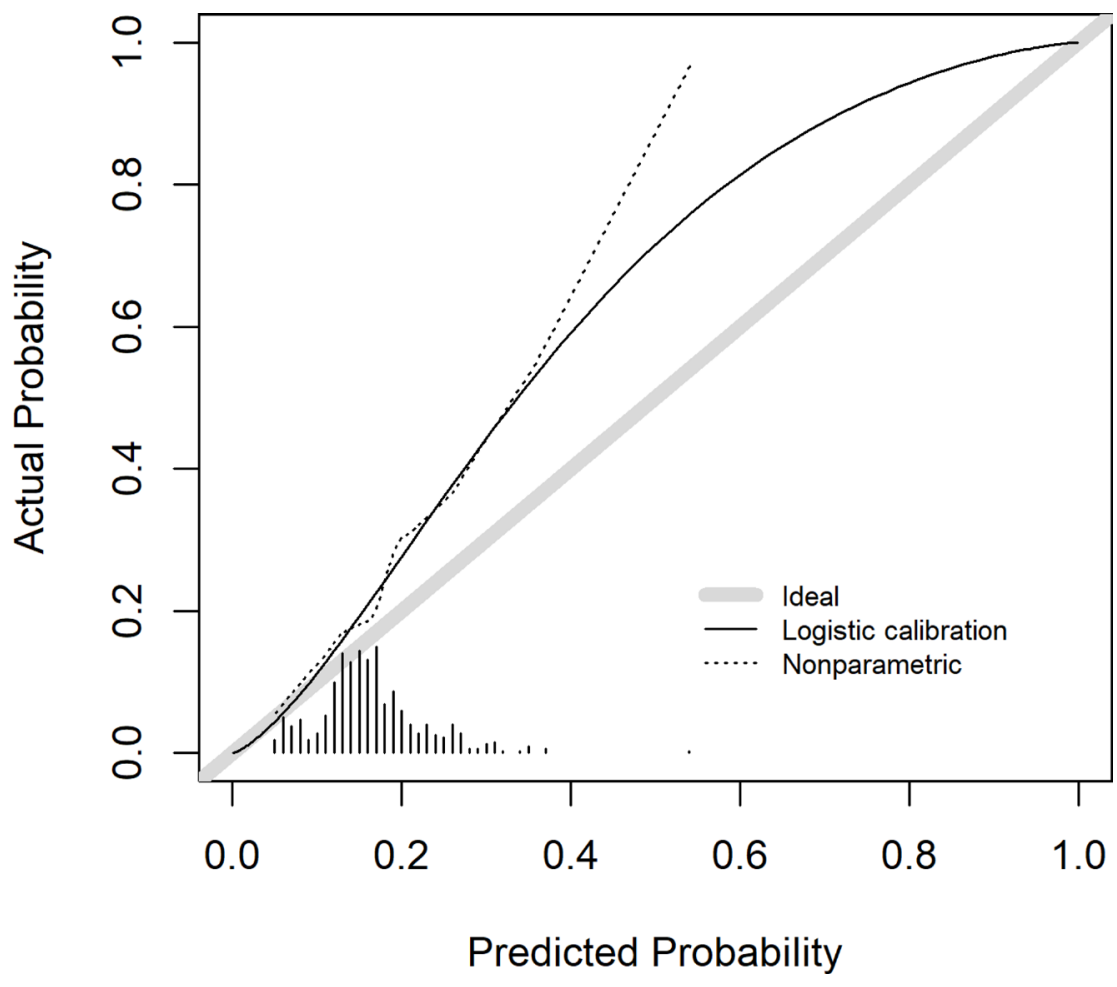

Figure 2: Calibration plot of PASS-RC validation in PIEM cohort. 
cores in Bx and PSA density, or Gleason score and PSA at baseline [29] to assess the risk of reclassification. The PASS Risk Calculator (PASS-RC) calculates the individual risk of reclassification in the follow up $\mathrm{Bx}$ of patients on AS $[14,15]$. The internal validation obtained an AUCROC of 0.72 , which bears acceptable discrimination ability. The authors elegantly claim for external validations to test the real capacity of this Risk Calculator before recommending its general implementation [15].

In this study we have analyzed the usefulness of the PASS-RC in the Spanish Registry on AS, with 1,024 AS patients included in our Registry at December

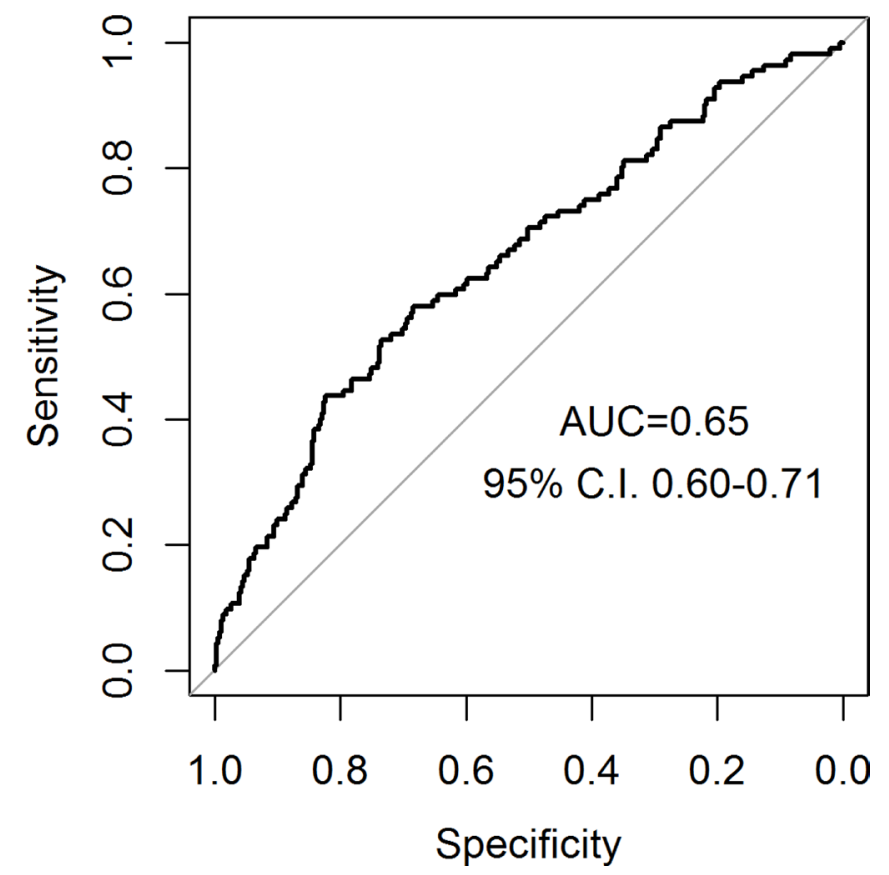

Figure 3: ROC curve of PASS-RC validation in PIEM cohort.

PASS risk calculator: Reclassification probabilities

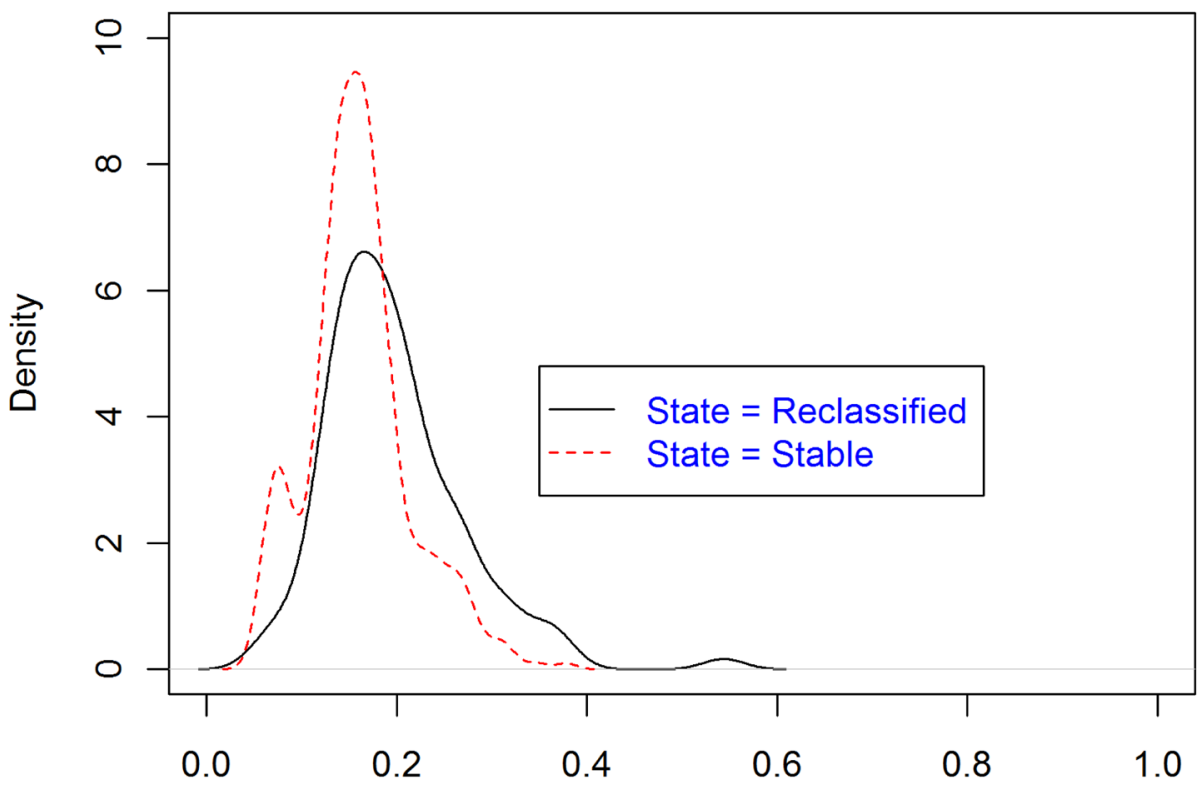

Threshold probability

Figure 4: Probability density functions of probability values obtained from PASS-RC in patients with/without reclassification in PIEM cohort. 
2015. This registry was opened at September 2014 and patients were collected prospective and retrospectively from several Spanish series with more evolution on AS; but most of them have been included at the beginning of their evolution on AS. This is the reason because only 498 patients have at least one follow-up Bx for the present validation. This fact could be considered a limitation, but in our opinion the more relevant follow-up Bx on AS is the confirmation $\mathrm{Bx}$, the $\mathrm{Bx}$ that certificate that the initial findings from the initial $\mathrm{Bx}$ are real and patients can continue on AS with a low risk of underclassification. So that we consider are validation focused on confirmation$\mathrm{Bx}$ specially relevant to validate the clinical useful of PASS-RC. As mentioned, no standardized protocol of follow up is defined in our Registry, so each center uses its own protocol and defines its own cohort as a real life clinical practice on AS, offering PASS-RC the opportunity to show its real utility in common clinical practice. Statistically, the differences between reference and study cohorts (Table 1), rather than representing a drawback, offer PASS-RC an adequate opportunity for external validation. Thus, our series of 498 patients is older than the PASS cohort and shows higher PSA levels than the PASS cohort, mainly in the interval $6-10 \mathrm{ng} / \mathrm{mL}$ and it is more conservative in $\mathrm{T}$ stage at diagnosis.

Regarding the results of the first follow up Bx, no cancer was found in $43.8 \%$ of the cases, compared to $36 \%$ in the PASS cohort $(p<0.01)$. In the following follow-up Bx this trend remained the same, without any significant difference. Reclassification rates during follow up Bx ranged between 10 and $22 \%$ in our series and between 20 and $30 \%$ in the reference series, without any statistical differences at any follow-up Bx between them (Figure 1). Therefore, we were able to unveil a trend to a lower reclassification rate in our series and a higher absence, or $\mathrm{PCa}$, at our follow up $\mathrm{Bx}$. To date we have been unable to assess if it would become statistically significant with a higher sample size in the future. Different diagnoses and AS strategies could have influenced these different outcomes. At the moment, the 2-month mean inferior interval between $\mathrm{Bx}$ in our cohort is the only objective justification for the lower detection of reclassification, particularly in cumulative follow-ups.

When we evaluated the correspondence between the predicted probabilities of the model in our validation series and the real incidence of reclassification, we observed how the model underestimates our reality of reclassification, mainly in the projection of high probabilities (Figure 2). In fact, the probabilities assigned by the model were low, ranging between 20 and 50\% (Figures 2 and 4), are coherent with a series which has a low risk of reclassification as the AS series are. The discrimination ability was lower in our series (AUC: 0.65 (Figure 3), which is common in external validations but compromises the clinical utility of the model.

\section{PASS risk calculator: Clinical utility curve}

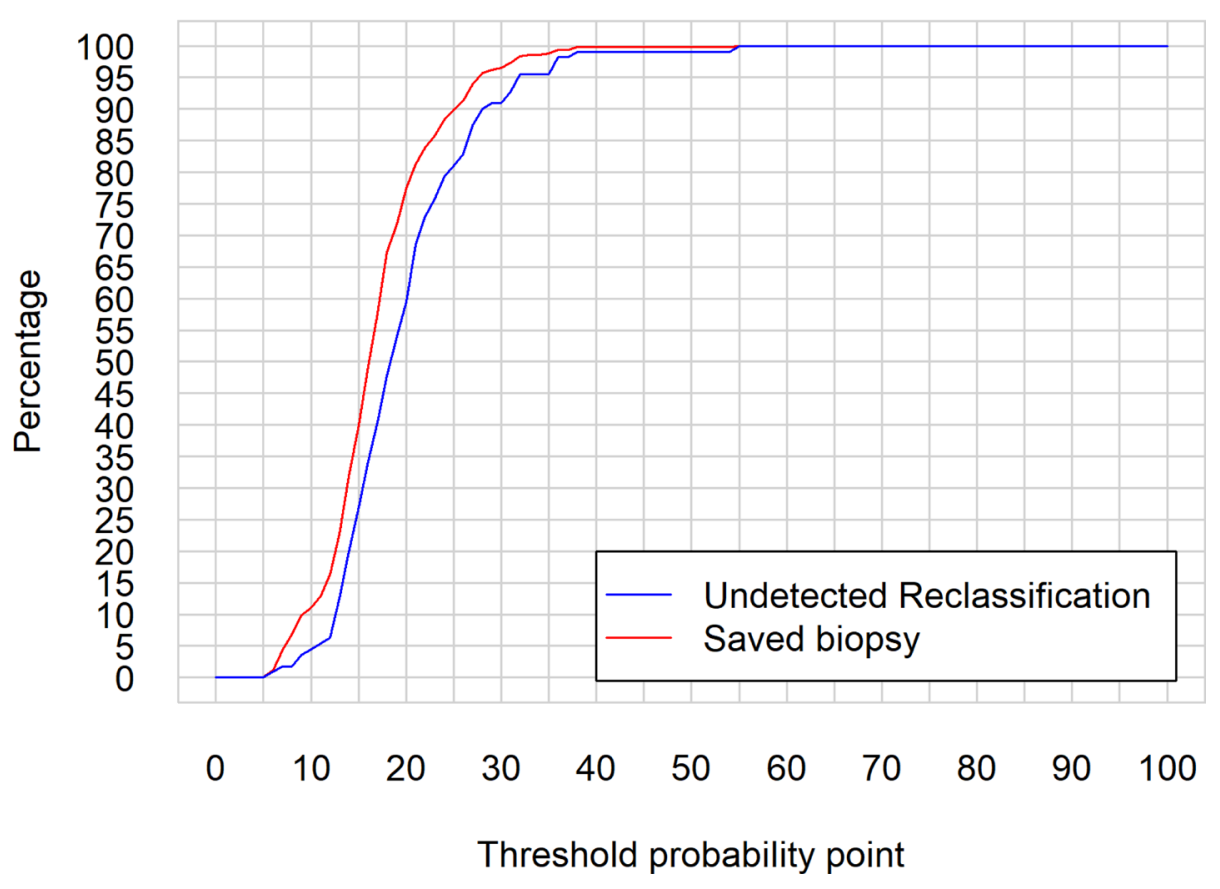

Figure 5: Clinical utility curve: For different threshold probability points selected in $X$ axe, it can be seen in the $Y$ axe, on the one hand, in blue line, the percentage of biopsies not performed to patients (Saved biopsy) and, in the other hand, in red line, the percentage of patients whose progression have been not been adequately diagnosed (Undetected reclassification). 
Table 3: Clinical utility cut-offs analysis

\begin{tabular}{lcc}
\hline Threshold (\%) & Saved biopsies (\%) & Undetected reclassifications (\%) \\
\hline 5 & 0 & 0 \\
6 & 1,21 & 0,9 \\
7 & 4,4 & 1,8 \\
8 & 6,8 & 1,8 \\
9 & 9,9 & 3,6 \\
10 & 11,1 & 4,5 \\
11 & 12,9 & 5,4 \\
12 & 16,3 & 6,3 \\
13 & 22,7 & 12,6 \\
14 & 31,8 & 19,8 \\
15 & 40,0 & 27,0 \\
16 & 49,3 & 34,2 \\
17 & 57,7 & 40,5 \\
18 & 67,4 & 47,7 \\
19 & 71,8 & 54,0 \\
20 & 77,5 & 59,5 \\
\hline
\end{tabular}

We also assessed the clinical utility of the model through probability density functions [30, 31]. We understand it is an excellent tool to choose cut-off points of clinical utility in predictive models, nomograms and risk calculators. Thus, in Figure 4 we can see the probabilities of reclassification provided by PASS-RC for both patients who progressed, or who did not, in the PIEM validation cohort. We expected higher probabilities of reclassification for patients who actually progressed but lower chances for the group of patients who did not.

From the distributions of probabilities analyzed, we tried to identify a cut-off which separated non-progressive patients (ideally, those with low probabilities assigned by the model and being under this cut-off) from trueprogressive patients (with assigned probabilities over this cut-off point), but we were not able to obtain conclusive results. Unfortunately, the assigned probabilities are extremely similar in their distributions between both subpopulations without the opportunity to identify a discriminative cut-off point (Figure 4).

Finally, in an attempt to maximize the evaluation of this PASS-RC tool we drew our proposal of clinical utility curves as previously [31] (Figure 5). We have shown how the range of probabilities for clinical decisions rises from

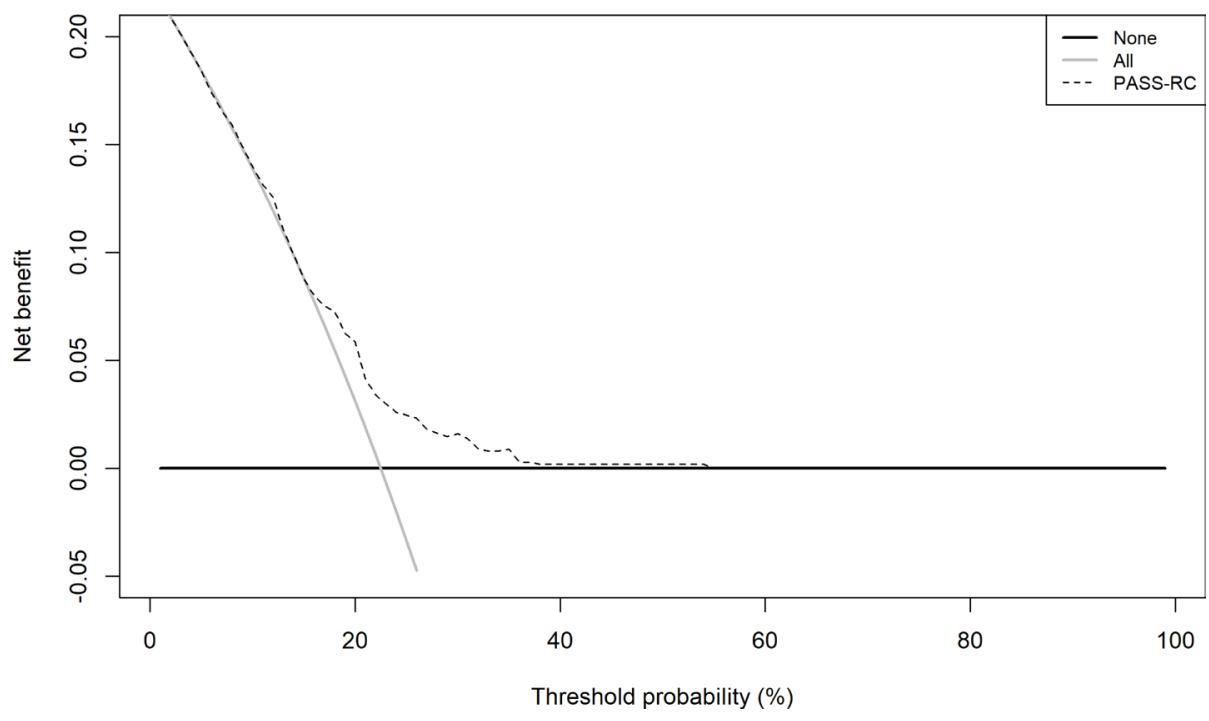

Figure 6: Decision curve analysis. 
Table 4: Combined models of PASS-RC analysis

\begin{tabular}{lcccccc}
\hline & \multicolumn{2}{c}{ Reclassification } & \multicolumn{2}{c}{ Reclassification } & AUC test \\
\cline { 2 - 7 } & $\boldsymbol{p}$-value & AUC & & $\boldsymbol{p}$-value & AUC & $\boldsymbol{p}$-value \\
\hline PASS-RC & $<.001$ & 0.654 & PASS-RC+ & & & \\
BMI $^{*}$ & 0.041 & 0.577 & BMI & 0.047 & 0.653 & 0.429 \\
PSAD & $<.001$ & 0.634 & PSAD & $<.001$ & 0.694 & 0.043 \\
Length $^{+}$ & 0.192 & 0.551 & Length & 0.062 & 0.655 & 0.933 \\
PCAi $^{++}$ & 0.582 & 0.512 & PCAi & 0.792 & 0.549 & 0.691 \\
\hline
\end{tabular}

BMI: body mass index. PSAD: PSA density. Length: PCa length in positive cores. PCAi: percentage of PCa involvement in biopsy. AUC: area under the ROC curve. BMI, Length and PCAi avalaible for ${ }^{*} n=238,{ }^{+} n=171 ;{ }^{+} n=176$.

$5 \%$ to $40 \%$. If we decided not to practice a follow-up Bx to patients with a reclassification probability under $13 \%$, we would save $23 \%$ of preplanned Bx, but would fail to detect $13 \%$ of the progressive patients in our common clinical practice of follow-up Bx, dangerous percentage of missing progressive patients especially in protocols where the follow-up biopsies are preplanned every three years after the confirmatory Bx.

We must acknowledge two main limitations in this paper. Firstly, the multicentricity and the lack of a standardized protocol of AS management in our entire cohort is a fruitful opportunity to externally validate PASS-RC in common clinical practice, but could have driven to the underdetection or misclassification of reclassification. A short follow-up can be mentioned as the second limitation of our study and more specifically the small sample size included in the last biopsies.

It is now clear that other known series of AS should validate the PASS-RC to confirm our findings, given that these clinical tools [32-33] are widely used in daily practice. As the authors suggest in their publication, we do agree that these tools have to be refined with time, using longer follow-up, and if possible, using the same common inexpensive variables. But they will probably be improved with new biomarkers [34-36] or morpho-functional parameters from mpMRI, as shown in other models [37]. In this sense, we have verified the improvement provided by PSA density. We strongly encourage the evaluation of this kind of clinical predictive tools - nomograms and risk calculators - using our proposed probability density functions and clinical utility curves, both in generation and validation cohorts. These graphs show the real classificatory accuracy of their predictions and help us to choose the best cut-off points for clinical use.

\section{CONCLUSIONS}

Using the Spanish multicentric registry study on AS as a validation cohort, we have obtained a moderate discrimination ability of PASS-RC but we found that it is not possible to choose a useful cut-off point to made adequate decisions in our clinical practice. Other external validations, the inclusion of new biomarkers, and especially the addition of morpho-functional parameters from mpMRI, could be implemented in future investigations to improve this model or to generate new ones.

\section{MATERIALS AND METHODS}

In the present study, we validate the PASS-RC as a predictive tool of the reclassification on follow-up $\mathrm{Bx}$ for patients on AS with one independent external series extracted from the AEU/PIEM/2014/0001 registry. We retrospectively and prospectively collected data from 1,024 patients in a multicentric study which included 36 Spanish hospitals.

The inclusion criteria were the same as those used for the PASS, namely a Gleason score $\leq 6$ and at least 10 cores on the initial Bx. In addition, the initial PSA value was below or equal to $20 \mathrm{ng} / \mathrm{ml}$. No unique followup protocol was determined for the AEU series, so every center scheduled their own AS protocol and it does not necessarily coincide with the PASS protocol. The outcome of our validation was reclassification (or disease upgrade), defined by the PASS-RC as either Gleason score upgrade from $\leq 6$ to $\geq 7$ and/or as an increase in percentage of cancer cores positive for cancer from $<34 \%$ to $\geq 34 \%$. A comparative descriptive analysis between PIEM and PASS cohorts at diagnosis was performed. In order to compare both cohorts, the age and PSA variables were categorized and statistical significant differences were calculated using chi-squared test (Table 1). We also included a comparison between both series regarding the data of the follow-up Bx (Table 2).

We evaluated the predictive accuracy of the PASS$\mathrm{RC}$ among our patients performing a standardized validation. Firstly, we obtained the probabilities estimated in the PIEM cohort using the PASS-RC [14, 15]. We drew the calibration curves, which evaluated the correspondence between the predicted and the actual probabilities of reclassification found in our cohort. Then, to study discrimination ability and the clinical utility of the model, the empirical distributions of probabilities of reclassification in the groups which actually reclassifies 
or not were graphically shown as the probability density functions (PDF) by kernel density estimation [38]. The overlap of the probability distributions of both populations was important to determine how the model discriminates between groups with and without the evaluated event. It also shows whether it was possible to choose a threshold for clinical application in order to split risk groups for reclassification. We proposed this PDF as a very useful way to choose the probability thresholds of clinical utility in previous validations of prostate cancer estimations [30, 31]. Moreover, discrimination was also quantified through the Receiver Operating Characteristics (ROC) curve [39], the area under the ROC curve (AUC) and its $95 \%$ confidence interval (CI). In order to estimate the saved biopsies and the reclassification delayed for different probabilities thresholds, we performed a graphical analysis through the Clinical utility curve (CUC) that we had previously designed to help this kind of clinical decisions [31]. Decision curve analysis was used to confirm the clinical utility analysis.

In addition, we explored, as other authors in previous studies [40, 41], the variables Body Mass index (BMI), PSA density, PCa length in positive cores and the percentage of $\mathrm{PCa}$ involvement as predictors of reclassifications, but here, in order to improve the discriminatory ability of PASS-RC. The AUC of PASS$\mathrm{RC}$ and the combination of the variables with PASS-RC were compared using the DeLong Test [42].

Statistical analyses were performed at the two-sided 0.05 significance level, using $\mathrm{R}$ programming language v.3.2.1 [43].

\section{Author contributions}

A Borque, J Rubio, LM Esteban: Protocol/ project development, Data collection or management, Data analysis, Manuscript writing/editing; A Collado, Y Pallás, PA López, J Huguet, JI Sanz, JM Gil, E Gómez, C Quicios, L Fumadó, S Martínez, J Soto: Protocol/project development, Data collection or management, Manuscript writing/editing.

\section{ACKNOWLEDGMENTS}

We would like to thank Concepción Orna for carefully reading this manuscript and Manolo Esparrago for his technical support. The authors thanks to all the investigators in the PIEM Active Surveillance Study Group: Herrera-Imbroda B., Duarte-Ojeda J.M., Celma-Domènech A., Congregado-Ruiz C.B., Montesino-Semper M., Hernández V., Clar-Blanch F., Torres-Roca M., PachecoBru J.J., Esquena S., Sánchez-Rodríguez C., GiménezBachs J.M., García-Rodríguez J.

\section{CONFLICTS OF INTEREST}

The authors declare that they have no conflict of interest.

\section{FUNDING}

None.

\section{REFERENCES}

1. Cózar JM, Miñana B, Gómez-Veiga F, RodríguezAntolín A, Villavicencio H, Cantalapiedra A, Pedrosa E. National prostate cancer registry 2010 in Spain. [Article in Spanish]. Actas Urol Esp. 2013; 37:12-9.

2. Rubio-Briones J, Borque A, Esteban LM, Iborra I, López PA, Gil JM, Pallás Y, Fumadó L, Martínez-Breijo S, Chantada V, Gómez E, Quicios C, Congregado CB, et al. Preliminary results of the Spanish Association of Urology National Registry in Active Surveillance for prostate cancer. Actas Urol Esp. 2016; 40:3-10.

3. Newcomb LF, Thompson IM Jr, Boyer HD, Brooks JD, Carroll PR, Cooperberg MR, Dash A, Ellis WJ, Fazli L, Feng Z, Gleave ME, Kunju P, Lance RS, et al. Outcomes of Active Surveillance for Clinically Localized Prostate Cancer in the Prospective, Multi-Institutional Canary PASS Cohort. J Urol. 2016; 195:313-20.

4. Klotz L, Zhang L, Lam A, Nam R, Mamedov A, Loblaw A. Clinical results of long-term follow-up of a large, active surveillance cohort with localized prostate cancer. J Clin Oncol. 2010; 28:126-31.

5. Dall'Era MA, Konety BR, Cowan JE, Shinohara K, Stauf F, Cooperberg MR, Meng MV, Kane CJ, Perez N, Master VA, Carroll PR. Active surveillance for the management of prostate cancer in a contemporary cohort. Cancer. 2008;112:2664-70.

6. van den Bergh RC, Roemeling S, Roobol MJ, Aus G, Hugosson J, Rannikko AS, Tammela TL, Bangma CH, Schröder FH. Outcomes of men with screen-detected prostate cancer eligible for active surveillance who were managed expectantly. Eur Urol. 2009; 55:1-8.

7. Soloway MS, Soloway CT, Eldefrawy A, Acosta K, Kava B, Manoharan M. Careful selection and close monitoring of low-risk prostate cancer patients on active surveillance minimizes the need for treatment. Eur Urol. 2010; 58:831-5.

8. Tosoian JJ, Trock BJ, Landis P, Feng Z, Epstein JI, Partin AW, Walsh PC, Carter HB. Active surveillance program for prostate cancer: an update of the Johns Hopkins experience. J Clin Oncol. 2011; 29:2185-90.

9. Adamy A, Yee DS, Matsushita K, Maschino A, Cronin A, Vickers A, Guillonneau B, Scardino PT, Eastham JA. Role of prostate specific antigen and immediate confirmatory biopsy in predicting progression during active surveillance for low risk prostate cancer. J Urol. 2011; 185:477-82. 
10. van As NJ, Norman AR, Thomas K, Khoo VS, Thompson A, Huddart RA, Horwich A, Dearnaley DP, Parker CC. Predicting the probability of deferred radical treatment for localised prostate cancer managed by active surveillance. Eur Urol. 2008; 54:1297-305.

11. Cussans A, Somani BK, Basarab A, Dudderidge T. The role of targeted prophylactic antimicrobial therapy prior to transrectal ultrasound (TRUS) guided prostate biopsy in reducing infection rates: a systematic review. BJU Int. 2016; 117:725-31.

12. Miocinovic R, Jones JS, Pujara AC, Klein EA, Stephenson AJ. Acceptance and durability of surveillance as a management choice in men with screen-detected, lowrisk prostate cancer: improved outcomes with stringent enrollment criteria. Urology. 2011; 77:980-4.

13. Ayres BE, Montgomery BS, Barber NJ, Pereira N, Langley SE, Denham P, Bott SR. The role of transperineal template prostate biopsies in restaging men with prostate cancer managed by active surveillance. BJU Int. 2012; 109:1170-6.

14. Available in: http://prostate-cancer-risk-calculator.org.

15. Ankerst DP, Xia J, Thompson IM Jr, Hoefler J, Newcomb LF, Brooks JD, Carroll PR, Ellis WJ, Gleave ME, Lance RS, Nelson PS, Wagner AA, Wei JT, et al. Precision Medicine in Active Surveillance for Prostate Cancer: Development of the Canary-Early Detection Research Network Active Surveillance Biopsy Risk Calculator. Eur Urol. 2015; 68:1083-8.

16. Goltz D, Holmes EE, Gevensleben H, Sailer V, Dietrich J, Jung M, Röhler M, Meller S, Ellinger J, Kristiansen G, Dietrich D. CXCL12 promoter methylation and PD-L1 expression as prognostic biomarkers in prostate cancer patients. Oncotarget. 2016; 7:53309-53320. https://doi. org/10.19632/oncotarget.10786.

17. Hansen AF, Sandsmark E, Rye MB, Wright AJ, Bertilsson H, Richardsen E, Viset T, Bofin AM, Angelsen A, Selnaes KM, Bathen TF, Tessem MB. Presence of TMPRSS2-ERG is associated with alterations of the metabolic profile in human prostate cancer. Oncotarget. 2016; 7:42071-42085. https://doi. org/10.19632/oncotarget.9817.

18. Ferro M, Lucarelli G, Bruzzese D, Di Lorenzo G, Perdonà $\mathrm{S}$, Autorino R, Cantiello $\mathrm{F}$, La Rocca R, Busetto GM, Cimmino A, Buonerba C, Battaglia M, Damiano R, et al. Low serum total testosterone level as a predictor of upstaging and upgrading in low-risk prostate cancer patients meeting the inclusion criteria for active surveillance. Oncotarget. 2017; 8:18424-18434. https://doi.org/10.19632/ oncotarget. 12906.

19. Ferro M, Ungaro P, Cimmino A, Lucarelli G, Busetto GM, Cantiello F, Damiano R, Terracciano D. Epigenetic Signature: A New Player as Predictor of Clinically Significant Prostate Cancer (PCa) in Patients on Active Surveillance (AS). Int J Mol Sci. 2017; 18:1146.

20. Cantiello F, Russo GI, Cicione A, Ferro M, Cimino S, Favilla V, Perdonà S, De Cobelli $\mathrm{O}$, Magno $\mathrm{C}$, Morgia $\mathrm{G}$,
Damiano R. PHI and PCA3 improve the prognostic performance of PRIAS and Epstein criteria in predicting insignificant prostate cancer in men eligible for active surveillance. World J Urol. 2016; 34:485-493.

21. de Cobelli O, Terracciano D, Tagliabue E, Raimondi S, Bottero D, Cioffi A, Jereczek-Fossa B, Petralia G, Cordima G, Almeida GL, Lucarelli G, Buonerba C, Matei DV, et al. Predicting pathological features at radical prostatectomy in patients with prostate cancer eligible for active surveillance by multiparametric magnetic resonance imaging. PLoS One. 2015; 10:e139696.

22. Klotz L, Vesprini D, Sethukavalan P, Jethava V, Zhang L, Jain S, Yamamoto T, Mamedov A, Loblaw A. Long-term follow-up of a large active surveillance cohort of patients with prostate cancer. J Clin Oncol. 2015; 33:272-7.

23. Rubio-Briones J, Borque Fernando Á. Responses and advisability of active surveillance in prostate cancer (in response to editorial comments by Dr. Sánchez Badajoz). Actas Urol Esp. 2016; 40:72-74.

24. Sánchez-de-Badajoz E. The inconveniences of active surveillance in prostate cancer. Actas Urol Esp. 2016; 40:69-71.

25. Tosoian JJ, Mamawala M, Epstein JI, Landis P, Wolf S, Trock BJ, Carter HB. Intermediate and Longer-Term Outcomes From a Prospective Active-Surveillance Program for Favorable-Risk Prostate Cancer. J Clin Oncol. 2015; 33:3379-85.

26. Lane JA, Donovan JL, Davis M, Walsh E, Dedman D, Down L, Turner EL, Mason MD, Metcalfe C, Peters TJ, Martin RM, Neal DE, Hamdy FC, ProtecT study group. Active monitoring, radical prostatectomy, or radiotherapy for localised prostate cancer: study design and diagnostic and baseline results of the ProtecT randomised phase 3 trial. Lancet Oncol. 2014; 15:1109-18.

27. Andriole GL, Crawford ED, Grubb RL 3rd, Buys SS, Chia D, Church TR, Fouad MN, Isaacs C, Kvale PA, Reding DJ, Weissfeld JL, Yokochi LA, O'Brien B, et al, PLCO Project Team. Prostate cancer screening in the randomized Prostate, Lung, Colorectal, and Ovarian Cancer Screening Trial: mortality results after 13 years of follow-up. J Natl Cancer Inst. 2012; 104:125-32.

28. Ross AE, Loeb S, Landis P, Partin AW, Epstein JI, Kettermann A, Feng Z, Carter HB, Walsh PC. Prostatespecific antigen kinetics during follow-up are an unreliable trigger for intervention in a prostate cancer surveillance program. J Clin Oncol. 2010; 28:2810-6.

29. Bul M, Zhu X, Valdagni R, Pickles T, Kakehi Y, Rannikko A, Bjartell A, van der Schoot DK, Cornel EB, Conti GN, Boevé ER, Staerman F, Vis-Maters JJ, et al. Active surveillance for low-risk prostate cancer worldwide: the PRIAS study. Eur Urol. 2013; 63:597-603.

30. Borque-Fernando Á, Esteban-Escaño LM, Rubio-Briones J, Lou-Mercadé AC, García-Ruiz R, Tejero-Sánchez A, Muñoz-Rivero MV, Cabañuz-Plo T, Alfaro-Torres J, Marquina-Ibáñez IM, Hakim-Alonso S, Mejía-Urbáez E, Gil-Fabra J, et al. A Preliminary Study of the Ability of the 4 Kscore test, the Prostate Cancer Prevention Trial-Risk 
Calculator and the European Research Screening ProstateRisk Calculator for Predicting High-Grade Prostate Cancer. Actas Urol Esp. 2016; 40:155-163.

31. Borque A, Rubio-Briones J, Esteban LM, Sanz G, Domínguez-Escrig J, Ramírez-Backhaus M, Calatrava A, Solsona E. Implementing the use of nomograms by choosing threshold points in predictive models: 2012 updated Partin Tables vs a European predictive nomogram for organ-confined disease in prostate cancer. BJU Int. 2014; 113:878-86.

32. Prostate Cancer Prevention Trial-Risk Calculator 2.0 [visited August 6, 2017]. Available in: http://deb.uthscsa. edu/URORiskCalc/Pages/calcs.jsp.

33. European Research Screening Prostate Cancer [visited August 6, 2017]. Available in: http://www.prostatecancerriskcalculator.com/.

34. 4Kscore Test - HUMS pilot study [visited August 6, 2017]. Available in: https://urostatisticalsolutions.shinyapps. io/4k_score/.

35. Hansen J, Auprich M, Ahyai SA, de la Taille A, van Poppel H, Marberger M, Stenzl A, Mulders PF, Huland H, Fisch M, Abbou CC, Schalken JA, Fradet Y, et al. Initial prostate biopsy: development and internal validation of a biopsyspecific nomogram based on the prostate cancer antigen 3 assay. Eur Urol. 2013; 63:201-9.

36. Rubio-Briones J, Borque A, Esteban LM, Casanova J, Fernandez-Serra A, Rubio L, Casanova-Salas I, Sanz G, Domínguez-Escrig J, Collado A, Gómez-Ferrer A, Iborra I, Ramírez-Backhaus $\mathrm{M}$, et al. Optimizing the clinical utility of PCA3 to diagnose prostate cancer in initial prostate biopsy. BMC Cancer. 2015; 15:633.
37. Schoots IG, Petrides N, Giganti F, Bokhorst LP, Rannikko A, Klotz L, Villers A, Hugosson J, Moore CM. Magnetic resonance imaging in active surveillance of prostate cancer: a systematic review. Eur Urol. 2015; 67:627-36.

38. Silverman B. Density Estimation for Statistics and data Analysis. Monogr Stat Appl Probab. 1986; 26:1-22.

39. Hanley JA, McNeil BJ. The meaning and use of the area under a receiver operating characteristic (ROC) curve. Radiology. 1982; 143:29-36.

40. de Cobelli O, Terracciano D, Tagliabue E, Raimondi S, Galasso G, Cioffi A, Cordima G, Musi G, Damiano R, Cantiello F, Detti S, Matei DV, Bottero D, et al. Body mass index was associated with upstaging and upgrading in patients with low-risk prostate cancer who met the inclusion criteria for active surveillance. Urologic Oncology: Seminars and Original Investigations. 2015; 33:201.e1-201.e8.

41. Russo GI, Cimino S, Castelli T, Favilla V, Urzì D, Veroux M, Madonia M, Morgia G. Percentage of cancer involvement in positive cores can predict unfavorable disease in men with low-risk prostate cancer but eligible for the prostate cancer international: active surveillance criteria. Urologic Oncology: Seminars and Original Investigations. 2014; 32:291-296.

42. DeLong ER, DeLong DM, Clarke-Pearson DL. Comparing the areas under two or more correlated receiver operating characteristic curves: a nonparametric approach. Biometrics. 1988; 44:837-845.

43. R Core Team. R: a language and environment for statistical computing. Vienna, Austria: R Foundation for Statistical Computing; 2016. http://www.R-project.org/. 